\title{
Anatomia comparada do lenho de Piptadenia gonoacantha (Mart.) J.F.Macbr. em dois tipos de vegetação
}

\author{
Eduardo Luiz Longui ${ }^{1,3}$, Alaor Bufolo ${ }^{2}$, Osny Tadeu de Aguiar ${ }^{1}$, Israel Luiz de Lima ${ }^{1}$ e \\ Sandra Monteiro Borges Florsheim ${ }^{1}$
}

Recebido: 16.04.2009; aceito: 09.12.2009

\begin{abstract}
Comparative wood anatomy of Piptadenia gonoacantha (Mart.) J.F.Macbr. in two vegetation types). Ecological anatomy aims to correlate the morphological and anatomical structures of plants with climatic, geographic and soil data. This study evaluated the qualitative and quantitative differences in the "pau-jacaré" wood - Piptadenia gonoacantha trees from Cantareira State Park (PEC) and Caetetus Ecological Station (EEC), respectively areas of Dense Ombrophylous Forest (Atlantic Forest) and Semi-deciduous Seasonal Forest. The anatomical parameters were analyzed according to usual techniques of wood anatomy and the data evaluated by analysis of variance (ANOVA) and multiple comparison tests. The results revealed statistically significant differences: trees from PEC had higher vessels frequency, lower, wider and more frequent rays, and fibers with larger diameters. In turn, trees from EEC showed higher vulnerability index and smaller pith vessels. The differences found in the wood specimens of the two sites could be due to variations in temperature, rainfall, altitude, latitude and longitude, although a larger number of individuals should be studied to determine possible ecological trends.
\end{abstract}

Key words: Piptadenia gonoacantha, wood anatomy, pau-jacaré

RESUMO - (Anatomia comparada do lenho de Piptadenia gonoacantha (Mart.) J.F.Macbr. em dois tipos de vegetação). A anatomia ecológica tem como objetivo correlacionar as estruturas morfológicas e anatômicas das plantas com dados climáticos, geográficos e do solo. Nesse contexto, avaliaram-se as diferenças qualitativas e quantitativas no lenho de árvores de pau-jacaré - Piptadenia gonoacantha procedentes do Parque Estadual Cantareira (PEC) e da Estação Ecológica de Caetetus (EEC), áreas respectivamente de Floresta ombrófila densa e Floresta estacional semidecidual. Os parâmetros anatômicos foram analisados seguindo técnicas usuais em anatomia de madeira e os dados avaliados por meio de análise de variância (ANOVA) e teste de comparações múltiplas. Os resultados revelaram diferenças estatisticamente significativas, sendo que indivíduos do PEC apresentaram maior frequência de vasos, raios mais baixos, largos e mais frequentes, além de fibras com maior diâmetro. Os indivíduos da EEC mostraram maior índice de vulnerabilidade e vasos com pontoações menores. As diferenças encontradas no lenho dos espécimes dos dois locais podem ser decorrentes de variações de temperatura, pluviosidade, altitude, latitude e longitude, embora um número maior de indivíduos deva ser analisado para se estabelecer possíveis tendências ecológicas.

Palavras-chave: Piptadenia gonoacantha, anatomia da madeira, pau-jacaré

\section{Introdução}

A anatomia ecológica tem como objetivo correlacionar as estruturas morfológicas e anatômicas das plantas com dados climáticos, geográficos e do solo e, apesar das folhas terem sido o principal foco de investigação da anatomia ecológica ao longo dos anos, há algumas décadas têm-se desenvolvido muitos trabalhos que avaliam a estrutura da madeira (Dickison 2000).
Podem ser citados alguns trabalhos que relacionaram a anatomia do lenho com diferentes condições latitudinais e tipos de vegetação, tais como os de: Van der Graaff \& Baas (1974), Carlquist (1975), Baas et al. (1983), Baas \& Carlquist (1985), BarajasMorales (1985), Carlquist \& Hoekman (1985), Ceccantini (1996), Alves \& Angyalossy-Alfonso (2000, 2002), Marcati et al. (2001) e Cosmo (2008).

De acordo com Metcalfe \& Chalk (1950) e Baas (1973), o ambiente interfere na morfologia e

1. Instituto Florestal, Caixa Postal 1322, 02377-000 São Paulo, SP, Brasil

2. Faculdades Oswaldo Cruz, R. Brigadeiro Galvão 540, 01151-000 São Paulo, SP, Brasil

3. Autor para correspondência: elongui@if.sp.gov.br 
anatomia das plantas, uma vez que o crescimento e desenvolvimento são influenciados por fatores como: disponibilidade hídrica, temperatura, características do solo e fatores geográficos; associados a estes há a genética e a fisiologia dos indivíduos, assim, fatores externos e internos somam-se originando a estrutura morfológica e anatômica da árvore (Fisher et al.2007).

De maneira geral, os trabalhos que investigam a variação no lenho em ambientes distintos mostram que as alterações quantitativas implicam em variações nas dimensões e frequência das células. Segundo AguilarRodríguez et al. (2006) e Cosmo (2008), muitos autores têm buscado identificar tendências gerais para determinadas floras, por meio da comparação entre plantas de ambientes xéricos e mésicos ou distribuídas em gradientes de latitude e/ou altitude. No entanto, são menos frequentes os estudos que investigam variações intraespecíficas do lenho, em resposta às variações ambientais, especialmente nas floras tropicais e subtropicais.

No Brasil, são exemplos os trabalhos de Alves \& Angyalossy-Alfonso $(2000,2002)$ que estudaram cerca de 490 espécies brasileiras e observaram variações anatômicas influenciadas pelas coordenadas geográficas. As autoras mencionaram que a latitude influenciou mais claramente a anatomia do lenho, especialmente as camadas de crescimento, características dos vasos e parênquima axial. Ceccantini (1996) em Casearia sylvestris do cerrado e Marcati et al. (2001) em Copaifera langsdorffii do cerradão encontraram vasos de maior diâmetro nesses ambientes em relação a espécimes encontrados em mata e floresta. Denardi \& Marchiori (2005), estudando Blepharocalyx salicifolius em dois locais diferentes quanto à pluviosidade, encontraram elementos de vaso mais longos, com maior diâmetro e menos frequentes em local com maior pluviosidade.

Devido à grande diversidade de espécies nas regiões tropicais, são necessários estudos para um melhor entendimento da influência das características ambientais na estrutura do lenho já que a mesma espécie ocorrendo em ambientes diferentes pode responder de forma distinta.

No estado de São Paulo há diversos tipos de vegetação, entre eles, a Floresta ombrófila densa e a Floresta estacional semidecidual, cada uma com características próprias, o que permite estudos comparativos com espécies que ocorrem naturalmente nessas duas formações florestais, como o pau-jacaré Piptadenia gonoacantha (Mart.) J.F.Macbr. Fabaceae (Leguminosae).
Além do estado de São Paulo, essa espécie ocorre naturalmente na Bahia, sul do Espírito Santo, Minas Gerais, Mato Grosso do Sul, leste e norte do Rio de Janeiro, Paraná e leste de Santa Catarina. $P$. gonoacantha é semicaducifólia podendo atingir quando adulta até $30 \mathrm{~m}$ de altura e $90 \mathrm{~cm}$ de DAP (diâmetro à altura do peito, 1,30 m do solo), seu tronco pode ser reto ou tortuoso com cristas aculeadas em toda a extensão. Possui cristas transversais e longitudinais na casca que lembram o couro de jacaré, daí seu nome popular. Quanto ao grupo sucessional, é uma espécie pioneira a secundária inicial, que cresce em vários tipos de solo. Embora a madeira não seja considerada de uso industrial é empregada para os mais diversos fins, como: brinquedos, embalagens, construção civil e acabamentos internos (Carvalho 2003).

O objetivo desse estudo foi identificar se há diferenças qualitativas e quantitativas no lenho de indivíduos de $P$. gonoacantha ocorrentes em dois tipos de vegetação: Floresta ombrófila densa e Floresta estacional semidecidual.

\section{Material e métodos}

Descrição das áreas experimentais - O estudo foi realizado em amostras do lenho de indivíduos ocorrentes em duas regiões diferentes do estado de São Paulo (figura 1). Parque Estadual Cantareira (PEC), área remanescente de Floresta ombrófila densa situada na zona norte da cidade de São Paulo, e municípios de Mairiporã, Caieiras e Guarulhos (Negreiros et al. 1974); e Estação Ecológica de Caetetus (EEC) localizada nos munícipios de Gália e Alvinlândia, área de Floresta estacional semidecidual (Tabanez et al. 2005, Ramos et al. 2008).

Alguns dados ambientais das áreas de estudo encontram-se na tabela 1.

Obtenção do material e análise anatômica - Por meio de método destrutivo foram retiradas amostras do lenho na altura do DAP (diâmetro a altura do peito $1,30 \mathrm{~cm}$ do solo) de três árvores de $P$. gonoacantha no Parque Estadual Cantareira (PEC), sendo considerada para este estudo apenas a porção do lenho próxima à casca. Amostras também no DAP de outras três árvores da mesma espécie foram obtidas por método não destrutivo, com auxílio de serrote, martelo e formão na Estação Ecológica de Caetetus (EEC). Todas as árvores foram identificadas no campo e as coordenadas geográficas obtidas por GPS, amostras das mesmas foram registradas e depositadas na Xiloteca do Instituto Florestal de São Paulo (SPSFw). 
Tabela 1. Informações dos tipos de vegetação, coordenadas geográficas e dados climáticos das áreas de estudo. Os valores de precipitação e temperatura para o PEC representam a média entre 1992 e 2007 . Os mesmos valores para a EEC representam a média de 1979 a 2001.

Table 1. Information on vegetation types, geographic coordinates and climatic data of studied areas. The precipitation and temperature values for PEC represent the average between 1992 and 2007. The same values for EEC represent the average of 1979 to 2001.

\begin{tabular}{lll}
\hline \multirow{2}{*}{ Dados ambientais } & \multicolumn{2}{c}{ Locais } \\
\cline { 2 - 3 } & PEC & EEC \\
\hline Tipo de vegetação & Floresta ombrófila densa & Floresta estacional semidecidual \\
Altitude $(\mathrm{m})$ & 1.150 & 660 \\
Latitude & $23^{\circ} 25^{\prime}$ & $22^{\circ} 22^{\prime}$ \\
Longitude & $46^{\circ} 40^{\circ}$ & $49^{\circ} 40^{\prime}$ \\
Precipitação média anual $(\mathrm{mm})$ & $* 1.320$ & $\# 1.430$ \\
Temperatura média anual $\left({ }^{\circ} \mathrm{C}\right)$ & $* 19,9^{\circ} \mathrm{C}$ & $\# 21,5^{\circ} \mathrm{C}$ \\
Clima (Sistema Köppen) & $*$ Cfb (temperado sem estação seca) & \#Cwa (quente com inverno seco) \\
\hline
\end{tabular}

* Faria et al. (dados não publicados); Faria et al. (unpublished data)

\# Tabanez et al. (2005); Tabanez et al. (2005)

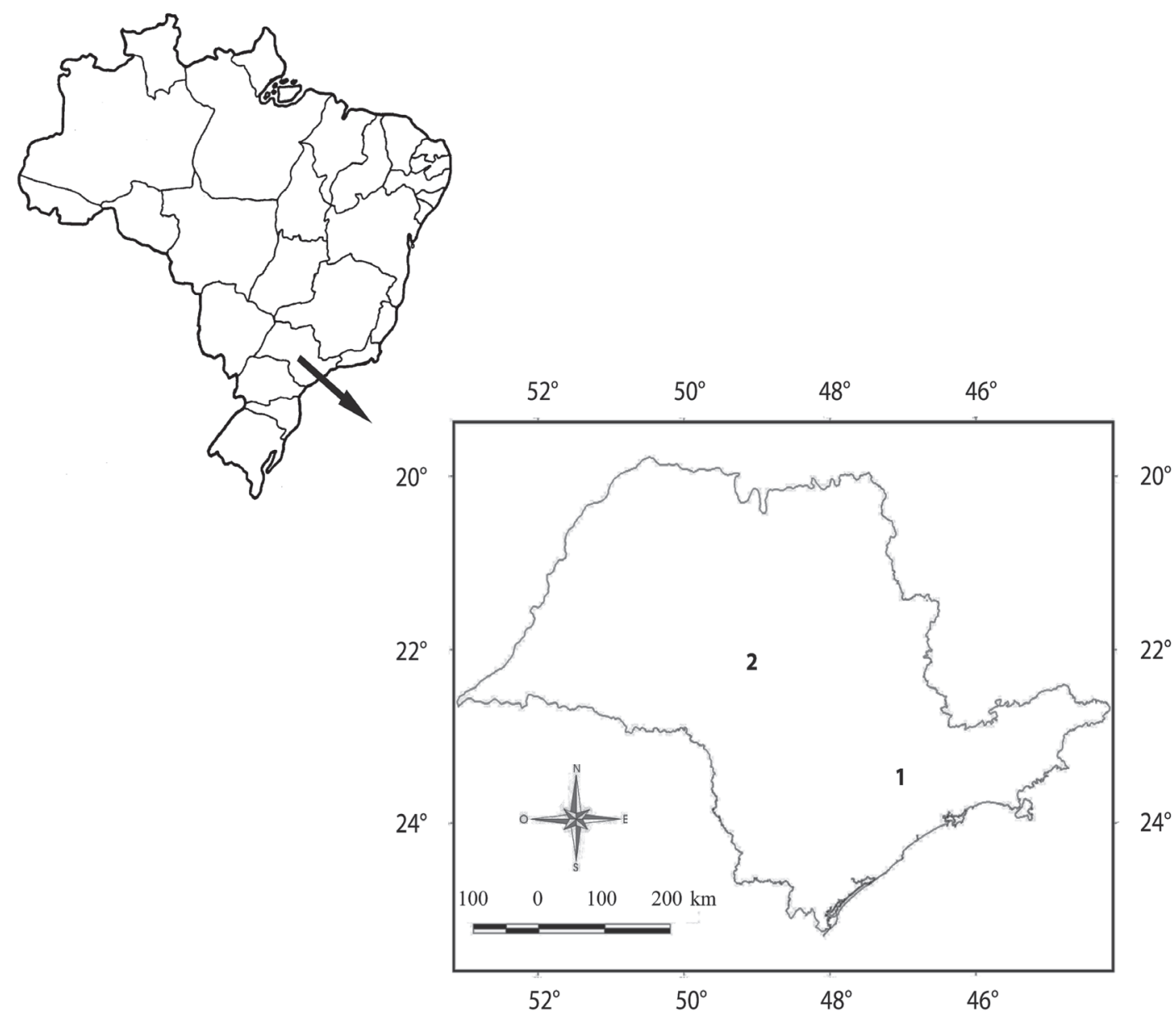

Figura 1. Localização das áreas de estudo. (1) Parque Estadual Cantareira (PEC), (2) Estação Ecológica de Caetetus (EEC).

Figure 1. Localization of studied areas. (1) Cantareira State Park(PEC), (2) Caetetus Ecological Station (EEC). 
Como não foi possível estabelecer a idade dos indivíduos analisados, buscou-se padronizar as coletas pelo diâmetro do DAP (tabela 2).

De cada amostra obtiveram-se corpos-de-prova com cerca de $2 \mathrm{~cm}^{3}$ e destes foram retirados pequenos fragmentos que foram macerados e posteriormente corados com safranina alcoólica a 1\% (Berlyn $\&$ Miksche 1976). Os corpos-de-prova foram amolecidos e seccionados em micrótomo de deslize Zeiss Hyrax S50, com espessura entre 12 e $25 \mu \mathrm{m}$. Secções de cada amostra foram clarificadas com hipoclorito de sódio a $60 \%$ e coradas com safranina e azul de astra 1\% (9:1) (Sass 1951). Prepararamse lâminas provisórias para a análise e mensuração das seguintes características anatômicas: diâmetro e frequência de vasos e comprimento dos elementos de vaso, diâmetro das pontoações intervasculares e raiovasculares; altura, largura e frequência dos raios; comprimento, diâmetro, lume e espessura da parede das fibras, de acordo com as recomendações do IAWA Committee (1989), sendo para cada característica adotado $n=25$, com exceção das pontoações, para as quais se adotou $n=10$. Também foi calculado o índice de vulnerabilidade (IV) de acordo com Carquist (1977).

Todas as mensurações foram realizadas em microscópios marca Olympus modelo BX 50 e CX 31, ambos equipados com software de análise de imagens Image - Pro Express versão 6.3.

Tabela 2. Dados das amostras de Piptadenia gonoacantha coletadas no Parque Estadual Cantareira (PEC) e Estação Experimental de Caetetus (EEC).

Table 2. Samples data of Piptadenia gonoacantha collected in Cantareira State Park (PEC) and Caetetus Ecological Station (EEC).

\begin{tabular}{lll}
\hline \multirow{2}{*}{ Locais } & \multicolumn{2}{c}{ Dados das amostras } \\
\cline { 2 - 3 } & SPSFw & DAP $(\mathrm{cm})$ \\
\hline \multirow{2}{*}{ PEC } & 3536 & 24 \\
& 3537 & 20 \\
& 3538 & 26 \\
EEC & 3539 & 30 \\
& 3540 & 20 \\
& 3541 & 25 \\
\hline
\end{tabular}

Análise estatística - A análise estatística dos resultados foi realizada com auxílio do programa SigmaStat 3.5 (SPSS Incorporation). Realizou-se análise estatística descritiva e a comparação entre as médias e desviospadrão para testar as diferenças entre os grupos. Com estes resultados, empregou-se o "t-test sample size" que confirmou ser suficiente o número de mensurações para atingir o poder desejado para o teste, de acordo com Eckblad (1991). Em seguida, realizou-se teste $\mathrm{T}$ para determinar quais características anatômicas, apresentaram variação significativa entre as amostras dos dois locais (tabela 2).

\section{Resultados}

Em todas as amostras, independente do local de coleta, observou-se a presença de camadas de crescimento demarcadas por fibras de parede mais espessa (figuras 2-5). Houve predomínio de vasos solitários, mais frequentes nas amostras da EEC (71\%) do que no PEC (62\%), sendo que a porcentagem de geminados e múltiplos de três e quatro foi respectivamente $19 \%$ e $9 \%$ na EEC e $23 \%$ e $-15 \%$ no PEC.

Nas amostras dos dois ambientes foram encontrados elementos de vaso com placa de perfuração simples, com apêndices em uma ou nas duas extremidades (figura 9), pontoações intervasculares alternas, arredondadas e guarnecidas (figura 8), pontoações raiovasculares semelhantes às intervasculares. Nas amostras do PEC observou-se conteúdo alaranjado nos vasos (figura 2).

Nas amostras dos dois ambientes constatou-se o predomínio de parênquima axial paratraqueal aliforme com cristais prismáticos (figura 10); raios homogêneos, multisseriados formados por células procumbentes (figuras $6,7,11$ ); ausência de estratificação; fibras libriformes, algumas visivelmente septadas, com pontoações simples. Máculas foram observadas nas amostras do PEC (Figura 12).

Para as características quantitativas observaramse diferenças estatisticamente significativas na frequência de vasos, altura, largura e frequência dos raios, diâmetro das fibras e índice de vulnerabilidade (tabela 3).

\section{Discussão}

Como se viu nos resultados, amostras coletadas no PEC e na EEC apresentaram camadas de crescimento demarcadas por fibras de parede mais espessa. 

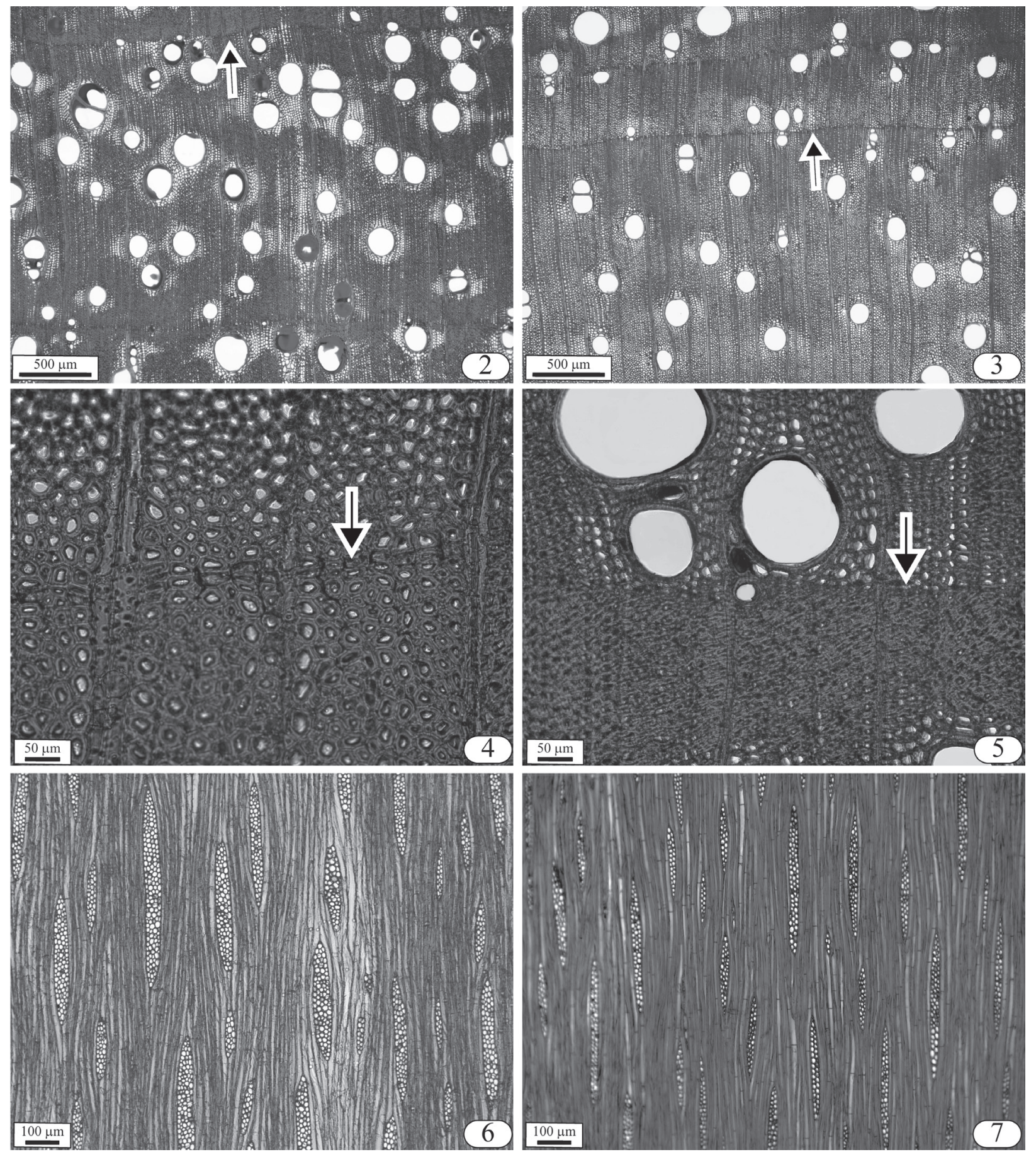

Figuras 2-5. Secções transversais. 2. Marcação da camada de crescimento (seta). 3. Notar a camada de crescimento (seta) e a variação na frequência de vasos em comparação com a figura anterior. 4-5. Destaque para a marcação de crescimento demarcada pela variação na espessura da parede das fibras (seta). 6-7. Secções longitudinais tangenciais, observar a variação entre a largura dos raios nos diferentes locais.

Figures 2-5. Transverse sections. 2. Growth layer (arrow). 3. Note the growth layer (arrow) and the variation in vessels frequency with respect to the previous figure and. 4-5. Highlight for marking of growth layer by variations in fibers wall thickness (arrow). 6-7. Tangential sections, note variation between the rays width in different sites. 
Tabela 3. Dados quantitativos do lenho de Piptadenia gonoacantha coletadas no Parque Estadual Cantareira (PEC) e Estação Experimental de Caetetus (EEC). Os valores são expressos em média e desvio padrão. Em cada linha, médias seguidas da mesma letra não diferem significativamente a $5 \%$ de probabilidade (teste $\mathrm{T}$ ). $\mathrm{n}=25, \mathrm{n}=10$ para as pontoações.

Table 3. Quantitative data of Piptadenia gonoacantha wood from Cantareira State Park (PEC) and from Caetetus Ecological Station (EEC). Values are expressed as mean and standard deviation. In each line, means followed by same letter do not differ significantly at $5 \%$ probability (T test). $\mathrm{n}=25, \mathrm{n}=10$ for pits.

\begin{tabular}{|c|c|c|c|c|}
\hline \multirow{2}{*}{ Características anatômicas } & \multicolumn{2}{|c|}{ Locais } & \multicolumn{2}{|c|}{ Teste $\mathbf{T}$} \\
\hline & PEC & EEC & $t$ & $P$ \\
\hline Diâmetro dos vasos (mm) & $139,4 \mathbf{a} \pm 35$ & $143,9 \mathbf{a} \pm 37,3$ & $-0,759$ & 0,449 \\
\hline Comprimento dos elementos de vaso (mm) & $298,3 \mathbf{a} \pm 69,5$ & $305,7 \mathbf{a} \pm 50,1$ & $-0,580$ & 0,563 \\
\hline Frequência dos vasos $\left(\mathrm{n}^{\circ} \mathrm{mm}^{-2}\right)$ & $9 \mathbf{a} \pm 3,4$ & $7 \mathbf{b} \pm 2,2$ & 3,475 & 0,001 \\
\hline Pontoações intervasculares (mm) & $5 \mathbf{a} \pm 0,4$ & $4,6 \mathbf{b} \pm 0,3$ & 2,929 & 0,005 \\
\hline Pontoações raiovasculares (mm) & $4,9 \mathbf{a} \pm 0,4$ & $4,6 \mathbf{b} \pm 0,4$ & 2,035 & 0,046 \\
\hline Índice de vulnerabilidade & $14,6 \mathbf{b} \pm 1,9$ & $21,5 \mathbf{a} \pm 4,1$ & $-6,089$ & 0,001 \\
\hline Altura dos raios $(\mathrm{mm})$ & $289,9 \mathbf{b} \pm 116,8$ & $338,7 \mathbf{a} \pm 101,9$ & $-2,437$ & 0,016 \\
\hline Largura dos raios (mm) & $36,7 \mathbf{a} \pm 9,1$ & $29,7 \mathbf{b} \pm 7,2$ & 4,669 & 0,001 \\
\hline Frequência dos raios $\left(\mathrm{n}^{\circ} \mathrm{mm}^{-1}\right)$ & $7 \mathbf{a} \pm 0,8$ & $5 \mathbf{b} \pm 1,1$ & 9,370 & 0,001 \\
\hline Comprimento das fibras (mm) & $909,7 \mathbf{a} \pm 142,0$ & $944,1 \mathbf{a} \pm 166,5$ & $-1,054$ & 0,295 \\
\hline Diâmetro das fibras (mm) & $20,2 \mathbf{a} \pm 3,9$ & $18,6 \mathbf{b} \pm 3,0$ & 2,178 & 0,032 \\
\hline Lume das fibras (mm) & $10,6 \mathbf{a} \pm 3,6$ & $9,2 \mathbf{a} \pm 3,6$ & 1,912 & 0,059 \\
\hline Espessura de parede das fibras (mm) & $4,7 \mathbf{a} \pm 1,0$ & $4,7 \mathbf{a} \pm 0,8$ & $-0,361$ & 0,719 \\
\hline
\end{tabular}

Segundo Fahn (1982) e Worbes (1989), a atividade cambial é fortemente influenciada por variações ambientais, como a temperatura e a disponibilidade de água, bem como por reguladores da própria planta. Lima et al. (2009) em estudo com E. contortisiliquum mencionam a presença de camadas de crescimento em plantas de floresta estacional semidecidual e caatinga, que possuem baixa disponibilidade de água durante certo período do ano. Reforçando essa idéia, Lisi et al. (2008) descrevem que o contraste de condições ambientais que ocorre no estado de São Paulo, com chuvas que ultrapassam $230 \mathrm{~mm}$ no verão e menos de $30 \mathrm{~mm}$ no inverno, influenciam na atividade cambial e, portanto, na resposta da planta ao ambiente.

Além das variações ambientais que ocorrem em um mesmo local ao longo do ano, devem ser consideradas as diferenças entre locais. No presente estudo, amostras de P. gonoacantha do PEC com menores médias anuais de pluviosidade e temperatura, apresentaram maior frequência de vasos, comprovada estatisticamente, com tendência a menor diâmetro e elementos mais curtos. O que está de acordo com Baas (1982) e Carlquist (1988), que descreveram a presença de vasos com menor diâmetro em ambientes mais secos ou frios, Denardi \& Marchiori (2005) estudando Blepharocalyx salicifolius em dois locais com disponibilidade hídrica diferentes, reportaram elementos de vaso mais curtos, com menor diâmetro e mais frequentes em local com menor pluviosidade; Wheeler et al. (2007) relacionam menor frequência de vasos com maior diâmetro a ambientes úmidos e menores latitudes, enquanto que vasos de menor diâmetro estão associados a maiores latitudes e ambientes com prolongados períodos de baixa disponibilidade hídrica.

A ocorrência de vasos solitários e múltiplos é comum na flora brasileira (Alves \& AngyalossyAlfonso 2000), mas as proporções podem variar de acordo com o ambiente. No presente estudo observouse menor proporção de vasos solitários e maior proporção de vasos geminados e múltiplos de três e quatro nas amostras do PEC, o que está de acordo com Carlquist \& Hoekman (1985) e Fahn et al. (1986) que relatam uma tendência a um maior agrupamento de vasos em ambientes com menor disponibilidade hídrica.

De acordo com Carlquist (1977), o índice de vulnerabilidade (razão entre o diâmetro e a frequência dos vasos) avalia a relação entre as características dos vasos e o ambiente, especificamente o risco da planta desenvolver interrupção da coluna de água por embolia, sendo que quanto menor o índice de 
vulnerabilidade, mais seguro é o xilema, sendo porém menos eficiente no transporte de água. No presente trabalho, as amostras do PEC, local de menor pluviosidade média, apresentaram índice de vulnerabilidade significativamente menor, o que pode expressar um ajuste às condições locais. $\mathrm{O}$ mesmo resultado foi obtido por Denardi \& Marchiori (2005) que encontraram menor índice de vulnerabilidade em local com menor disponibilidade hídrica.

Neste estudo, constatou-se menores pontoações intervasculares e raiovasculares nas amostras da EEC, local com maior pluviosidade média, resultado contrário do observado por Marcati et al. (2001) em Copaifera langsdorffii que encontraram pontoações intervasculares maiores em indivíduos de Floresta com relação àqueles de Cerradão; e Luchi (2004) em Croton urucurana em solos com diferentes níveis de umidade, que encontrou pontoações de maior diâmetro em espécimes de área alagada. Zimmermann (1982), Baas et al. (1983), Barajas-Morales (1985) e Lindorf (1994), descrevem que pontoações intervasculares pequenas consistem em uma estratégia para aumentar a segurança no lenho, segundo Wheeler et al. (2007), pontoações de maiores dimensões, possuem maiores aberturas que aumentam os riscos de embolia.

As correlações entre o ambiente e características dos vasos são esperadas, pois estas são as células que conduzem a água, um fator determinante para as variações no lenho. No entanto, há poucos estudos que correlacionaram as características dos raios
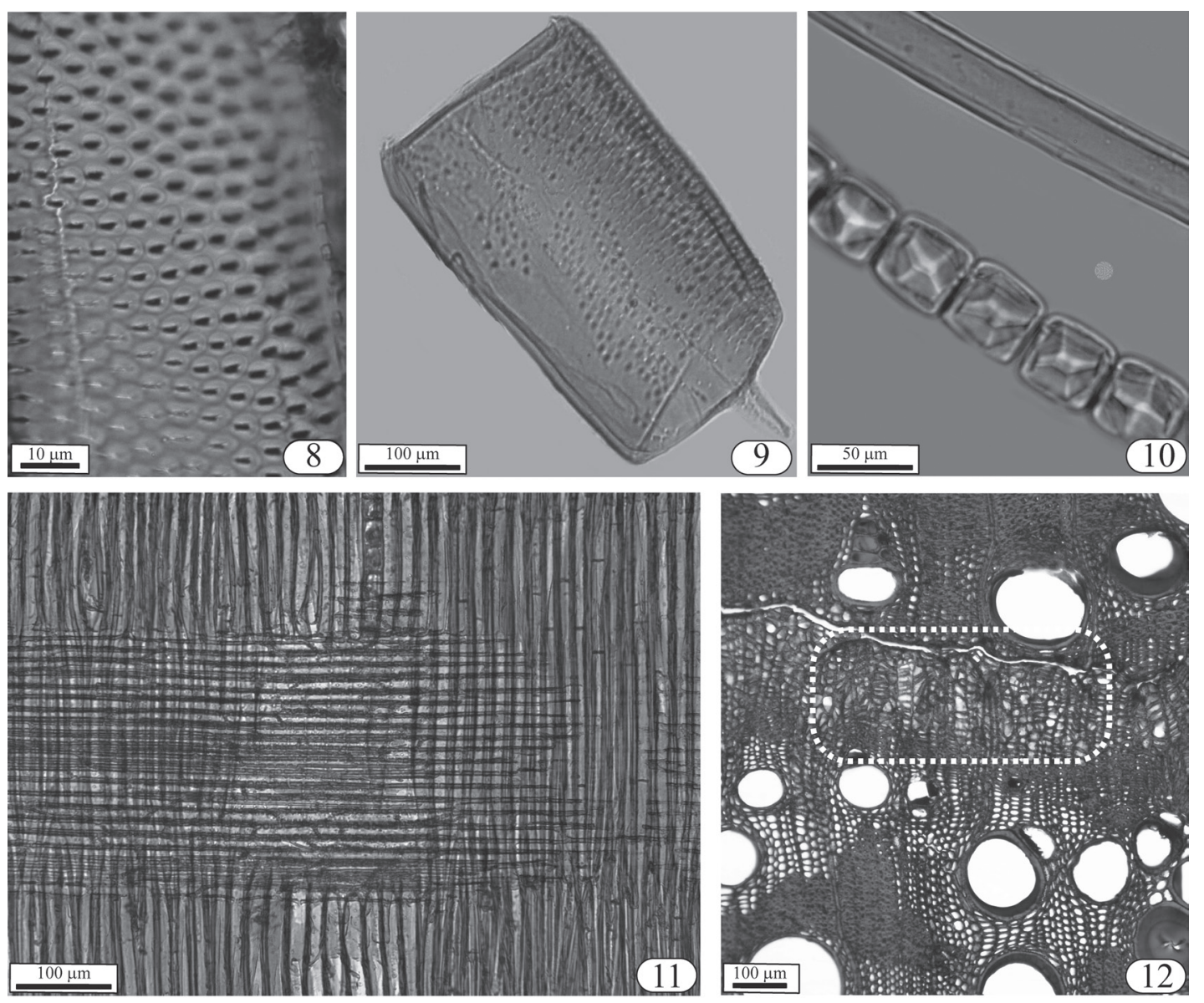

Figuras 8-10. Lenho dissociado. 8. Detalhe de pontoação intervascular alterna. 9. Elemento de vaso com apêndice em uma extremidade. 10. Sequência de células parenquimáticas formando série cristalífera. 11. Secção longitudinal radial, notar raio homogêneo com células procumbentes. 12. Secção transversal de amostra do PEC com destaque para mácula medular (retângulo).

Figures 8-10. Macerated tissue. 8. Detail of intervessel pits alternate. 9. Vessel element with a tail at one end. 10. Crystals in parenchyma cells. 11. Radial section, note all ray cells procumbent. 12. Transverse section of PEC sample with pith fleck (rectangle). 
com o ambiente. Em $P$. gonoacantha observou-se raios significativamente mais baixos, largos e mais frequentes nas amostras do PEC, local com maior altitude e latitude, menor precipitação e menor temperatura média em relação às amostras da EEC. Lima et al. (2009) também observaram raios mais largos em indivíduos de local mais seco. Em solos com diferentes níveis de umidade, Luchi (2004) observou raios mais largos em Croton urucurana coletado em solo mais seco. Luchi et al. (2005) mencionam que há indícios de que espécies com raios mais largos estão sujeitas a um ambiente hidricamente desfavorável. Segundo Alves \& Angyalossy-Alfonso (2002) raios mais largos em espécies que vivem em clima sazonal pode melhorar o transporte de metabólitos do floema para o xilema e suprir o câmbio no início da estação mais favorável ao crescimento.

Da mesma forma que os raios, as fibras também são pouco exploradas em trabalhos de anatomia ecológica. Neste estudo, foi observada variação significativa apenas no diâmetro das fibras, maior nas amostras do PEC, embora se tenha observado uma tendência de fibras mais curtas nas amostras do PEC. Resultados semelhantes foram encontrados por Noshiro \& Baas (2000) e Denardi \& Marchiori (2005) que mencionaram a ocorrência de fibras mais curtas em ambiente com menor precipitação. Já Ribeiro e Barros (2006) em Pseudopiptadenia contorta observaram fibras mais longas em local com maior altitude, além de menor precipitação e temperatura; Liu \& Noshiro (2003) encontraram correlação positiva entre o comprimento da fibra e a altitude em Dodonaea viscosa. Como as variações nas fibras não conferem uma vantagem ecológica evidente, uma vez que a sua função fundamental é a sustentação e não o transporte de água (Alves \& Angyalossy-Alfonso 2002) é difícil estabelecer tendências ecológicas nessas células, principalmente porque há muita variação em suas dimensões nas direções radial e axial (Lima et al. 2009).

Quando se pretende avaliar variações no lenho decorrentes do ambiente, devem ser considerados, além da precipitação, outros fatores ambientais como características do solo, sua permeabilidade e capacidade de retenção de água, entre outros. Vários autores correlacionaram alterações no lenho com as variações geográficas; Liu \& Noshiro (2003), avaliando espécimes de Dodonaea viscosa em diferentes altitudes na China e Japão, encontraram fibras e elementos de vaso mais longos, além de maior frequência de vasos em maiores altitudes. Noshiro \&
Baas (2000) para Cornus spp. e Fisher et al. (2007) em Metrosideros polymorpha observaram vasos de menor diâmetro em maiores altitudes, Van der Graaff \& Baas (1974) mencionam que entre outras características do lenho, o diâmetro dos vasos e o comprimento dos elementos de vaso tendem a diminuir com o aumento da latitude. Marcati et al. (2001) para Copaifera langsdorffii também encontraram vasos de maior diâmetro em maior latitude.

Quando se analisa os dados geográficos dos locais de estudo, verifica-se que o PEC apresenta maior altitude e latitude; nas amostras coletadas nesse local há variações significativas, com maior frequência de vasos e fibras de maior diâmetro.

Conclui-se que nas amostras de $P$. gonoacantha estudadas, características dos vasos como o diâmetro e comprimento, explorados em outros trabalhos de anatomia ecológica, não mostraram variações conclusivas. Já a frequência dessas células apresentou diferenças significativas, o que pode estar relacionado com as condições ambientais, especialmente a pluviosidade. Para os raios foram encontradas diferenças estatisticamente significativas em todas as características avaliadas - altura, largura e frequência - diferenças que podem estar ligadas à sazonalidade. Embora as fibras tenham mostrado variação significativa em seus diâmetros, esses resultados não mostram uma relação clara com as variações ambientais. Considerando o número de indivíduos analisados, não é possível propor tendências ecológicas para $P$. gonoacantha, no entanto, quando se avaliam comparativamente o lenho dos espécimes dos dois locais, observam-se diferenças que podem estar ligadas às variações de temperatura, pluviosidade, altitude e latitude.

\section{Literatura citada}

Aguilar-Rodríguez, S., Terrazas, T.\& López-Mata, L. 2006. Anatomical wood variation of Buddleja cordata (Buddlejaceae) along its natural range in Mexico. Trees 20: 253-261.

Alves, E.S. \& Angyalossy-Alfonso, V. 2000. Ecological trends in the wood anatomy of some Brazilian species. I: Growth rings and vessels. IAWA Journal 21: 3-30.

Alves, E.S. \& Angyalossy-Alfonso, V. 2002. Ecological trends in the wood anatomy of some Brazilian species. 2. Axial parenchyma, rays and fibres. IAWA Journal 23: 391-418.

Baas, P. 1973. The wood anatomical range in Ilex (Aquifoliaceae) and its ecological and phylogenetic significance. Blumea 21: 193-258. 
Baas, P. 1982. Systematic, phylogenetic, and ecological wood anatomy - History and perspectives. In: P. Baas (ed.). New perspectives in wood anatomy. Junk, Nijhoff \& Hingham, The Hague, pp. 23-58.

Baas, P. \& Carlquist, S. 1985. A comparison of the ecological wood anatomy of the floras of southern California and Israel. IAWA Bulletin 6: 349-353.

Baas, P., Wheeler, E. \& Fahn, A. 1983. Some ecological trends in vessel characters. IAWA Bulletin 4: 141-159.

Baas, P. \& Schweingruber, F.H. 1987. Ecological trends in the wood anatomy of trees, shrubs

and climbers from Europe. IAWA Bulletin 8: 245-274.

Barajas-Morales, J. 1985. Wood structural differences between trees of two tropical forests in México. IAWA Bulletin 6: 355-364.

Berlyn, G.P. \& Miksche, J.P. 1976. Botanical microtechnique and cytochemistry. The Iowa University Press, Iowa.

Carlquist, S. 1975. Ecological strategies in xylem evolution. University of California Press, Berkeley.

Carlquist, S. 1977. Ecological factors in wood evolution: a floristic approach. American Journal of Botany 64: 887-896.

Carlquist, S. 1988. Comparative wood anatomy: systematic, ecological and evolutionary aspects of dicotyledons wood. Springer Verlag, Berlin.

Carlquist, S. \& Hoekman, D.A. 1985. Ecological wood anatomy of the woody Southern Californian flora. IAWA Bulletin 6: 319-347.

Carvalho, P.E.R. 2003. Espécies Arbóreas Brasileiras. Brasília: Embrapa Informação Tecnológica; Embrapa Florestas, Colombo.

Ceccantini, G. 1996. Anatomia ecológica do lenho de espécies de cerrado e mata: Casearia sylvestris Sw. e Machaerium villosum Vog. Dissertação de Mestrado, Universidade de São Paulo, São Paulo.

Cosmo, N.L. 2008. Anatomia ecológica e crescimento do lenho de Sebastiania commersoniana (Baillon) Smith e Downs, em diferentes condiçőes geomorfológicas e pedológicas da planície do Rio Iguaçu, PR, Brasil. Dissertação de Mestrado, Universidade Federal do Paraná. Curitiba.

Denardi, L.\& Marchiori, J.N.C. 2005. Anatomia ecológica da madeira de Blepharocalyx salicifolius (H.B.K.) Berg. Ciência Florestal 15: 119-127.

Dickison, W.C. 2000. Integrative plant anatomy. Academic Press, San Diego.

Eckblad, J.W. 1991. How many samples should be taken. Bio Science 41: 346-348.

Fanh, A. 1982. Plant Anatomy. 3 ed. Pergamon Press, Madrid.

Fahn, A., E. Werker \& P. Baas. 1986. Wood anatomy and identification of trees and shrubs from Israel and adjacent regions. The Israel Acad. of Sciences and Humanities, Jerusalem.

Fisher, J.B., Goldstein, G., Jones, T.J. \& Cordell, S. 2007. Wood vessel diameter is related to elevation and genotype in the Hawaiian tree Metrosideros polymorpha (Myrtaceae). American Journal of Botany 94: 709-715.

Iawa Committee. 1989. IAWA list of microscopic features for hardwood identification. IAWA Bulletin 10: 219332.

Lima, R.S., Oliveira, P., \& Rodrigues, L.R. 2009. Anatomia do lenho de Enterolobium contortisiliquum (Vell.) Morong (Leguminosae-Mimosoideae) ocorrente em dois ambientes. Revista Brasileira de Botânica 32: 361-374.

Lindorf, H. 1994. Eco-anatomical wood features of species from a very dry tropical forest. IAWA Journal 15: 361-376.

Lisi, C.S., Tomazello, M., Botosso, P.C., Roig, F.A., Maria, V.R.B., Ferreira-Fedele, L.\& Voigt, A.R.A. 2008. Tree-ring formation, radial increment periodicity, and phenology of tree species from a seasonal semideciduous forest in Southeast Brazil IAWA Journal 29: 189-207.

Liu, J. \& Noshiro, S. 2003. Lack of latitudinal trends in wood anatomy of Dodonaea viscosa (Sapindaceae), a species with a worldwide distribution. American Journal of Botany 90: 532-539.

Luchi,A.E. 2004. Anatomia do lenho de Croton urucurana Baill. (Euphorbiaceae) de solos com diferentes níveis de umidade. Revista Brasileira de Botânica 27: 271-280.

Luchi, A.E., Silva, L.C.P. \& Moraes, M.A. 2005. Anatomia comparada do lenho de Xylopia aromatica (Lam.) Mart. em áreas de cerrado e de plantações de Pinus elliottii Engelm. Revista Brasileira de Botânica 28: 809-820.

Marcati, C.R., Angyalossy-Alfonso, V. \& Benetati, L. 2001. Anatomia comparada do lenho de Copaifera langsdorffii Desf. (Leguminosae-Caesalpinoideae) de floresta e cerradão. Revista Brasileira de Botânica 24: 311-320.

Metcalfe, C. \& Chalk, L. 1950. Anatomy of the Dicotyledons. v.2. Claredon Press, Oxford.

Negreiros, O.C., Carvalho, C.T., Cesar, S.F, Duarte, F.R., Deshler, W.O.\& Thelen, K.D. 1974. Plano de Manejo para o Parque Estadual da Cantareira. Boletim Técnico n.10.

Noshiro, S. \& Baas, P. 2000. Latitudinal trends in wood anatomy within species and genera: case study in Cornus s.l. (Cornaceae). American Journal of Botany 87: 1495-1506.

Ramos, V.S., Durigan, G., Franco, G.A.D.C., Siqueira, M.F. \& Rodrigues, R.R. 2008. Árvores da floresta estacional semidecidual: Guia de Identificação de Espécies. EDUSP, São Paulo. 
Ribeiro, M.L.R.C. \& Barros, C.F. 2006. Variação intraspecífica do lenho de Pseudopiptadenia contorta (DC.) G.P. Lewis \& M.P. Lima (Leguminosae Mimosoideae) de populações ocorrentes em dois remanescentes de Floresta Atlântica. Acta Botanica Brasilica 20: 839-844.

Sass, J.E. 1951. Botanical microtechniche. The Iowa State College Press, Ames.

Tabanez, M.F., Durigan, G., Keuroghlian, A., Babosa, A.F., Freitas, C.A, Silva, C.E.F., Silva, D.A., Eaton, D.P., Brisolla, G., Faria, H.H., Mattos, I.F.A., Lobo, M.T., Barbosa, M.R., Rossi, M., Souza, M.G., Machado, R.B., Pfeifer, R.M., Ramos, V.S., Andrade, W.J., Contieri, W.A. \& Delgado, J.M. 2005. Plano de Manejo da Estação Ecológica dos Caetetus. IF Série Registros 29: 1-104.
Van Der Graaff, N.A. \& Baas, P. 1974. Wood anatomical variation in relation to latitude and altitude. Blumea 22: 101-121.

Wheeler, E.A. \& Baas, P. 1991. A survey of the fossil record for dicotyledonous wood and its significance for evolutionary and ecological wood anatomy. IAWA Bulletin 12: 275-332

Wheeler, E.A. \& Baas, P., Rodgers, S. 2007. Variations in dicot wood anatomy: a global analysis based on the insidewood database. IAWA Journal 28: 229-258.

Worbes, M. 1989. Growth rings, increment and age of trees in inundation forests, savannas and a mountain forest in the Neotropics. IAWA Bulletin 10: 109-122.

Zimmermann, M.H. 1982. Functional xylem anatomy of angiosperms trees. In: P. Baas (ed.). New perspectives in wood anatomy. Martinus Nijhoff Publishers, Boston. 